\title{
Contrast enhanced MRA with CAPR (Cartesian Acquisition with Projection like Reconstruction) technique - review of initial clinical experience
}

\author{
Manoharan Muthuvelu" ${ }^{1 *}$, Philip M Young ${ }^{1}$, Paul T Weavers², Stephen J Riederer ${ }^{2}$ \\ From 17th Annual SCMR Scientific Sessions \\ New Orleans, LA, USA. 16-19 January 2014
}

\section{Background}

Contrast enhanced MRA is a well validated and widely used technique for noninvasive vascular imaging. Significant advances in coil development, parallel imaging, and view-sharing techniques can enable imaging with very high spatial and/or temporal resolution which can be tailored to very specific or problematic clinical situations. Cartesian acquisition with projection like reconstruction (CAPR) is a specific technique developed by our MR Research laboratory which supports very high acceleration factors and has been adopted into clinical practice for a number of clinical scenarios. We will present a brief introduction to the technique and an illustrative review of our early clinical experience using CAPR for clinical imaging.

\section{Methods}

CAPR apportions the Cartesian phase-encoded $k Y-k Z$ plane into a low-spatial-frequency center region and a high-spatial-frequency outer annulus. The annulus is further divided into sets of projection-like vanes. The corners of the $\mathrm{kY}-\mathrm{kZ}$ plane are not sampled and are zero-filled, and data for the unsampled gaps between vanes are estimated by $2 \mathrm{D}$ homodyne processing. An individual image update consists of elliptical-centric sampling of the center region and one annular vane set, with view sharing applied from previous samples of the other vane sets. This vane pattern gives a visual appearance similar to projection reconstruction. Alternate vanes are sampled or not sampled, allowing for $2 \mathrm{D}$ homodyne detection to be performed across the $\mathrm{kY}-\mathrm{kZ}$ plane. Because homodyne detection is performed in this plane, full echoes are sampled along the readout $(\mathrm{kX})$ direction.

\section{Results}

We have used this technique in both single station and multi-station implementations, the latter using a novel technique of "fluoro-tracking" enabled by real-time reconstruction of image data to allow interactive decision making by the technologist to track the passage of a contrast bolus through multiple stations. We will present a number of cases illustrating the use, including imaging of arteriovenous malformations, imaging peripheral vascular runoff to facilitate bypass graft planning the setting of failed prior CTA, and imaging of the small vessels of the hands and feet.

\section{Conclusions}

CAPR is a novel and developing MRA technique which can exploit very high spatial and temporal resolution, and early implementation in our practice has shown promising results.

\section{Funding}

None.

\section{Authors' details}

'Radiology, Mayo Clinic, Rochester, Minnesota, USA. ${ }^{2}$ MR Research Laoratory, Mayo Clinic, Rochester, Minnesota, USA.

Published: 16 January 2014

doi:10.1186/1532-429X-16-S1-077

Cite this article as: Muthuvelu et al: Contrast enhanced MRA with CAPR

(Cartesian Acquisition with Projection like Reconstruction) technique review of initial clinical experience. Journal of Cardiovascular Magnetic Resonance 2014 16(Suppl 1):077.

${ }^{1}$ Radiology, Mayo Clinic, Rochester, Minnesota, USA

Full list of author information is available at the end of the article 